\title{
ON THE SPECTRAL SINGULARITIES AND SPECTRALITY OF THE HILL OPERATOR
}

\author{
O. A. VELIEV
}

Abstract. First we study the spectral singularity at infinity and investigate the connections of the spectral singularities and the spectrality of the Hill operator. Then we consider the spectral expansion when there is not the spectral singularity at infinity.

Mathematics subject classification (2010): 34L05, 34L20.

Keywords and phrases: Hill operator, spectral singularities, spectral operator, spectral expansion.

\section{REFERENCES}

[1] M. S. P. Eastham, The Spectral Theory of Periodic Differential Equations, Scottish Academic Press, Edinburgh, 1973.

[2] I. M. GELFAND, Expansions in series of eigenfunctions of an equation with periodic coefficients, Soviet Math. Dokl. 73 (1950), 1117-1120.

[3] F. GESZTESY AND V. TKACHENKO, When is a non-self-adjoint Hill operator a spectral operator of scalar type, C. R. Acad. Sci. Paris, Ser. I, 343 (2006) 239-242.

[4] F. GESZTESY AND V. TKACHENKO, A criterion for Hill operators to be spectral operators of scalar type, J. Analyse Math. 107 (2009) 287-353.

[5] D. McGarveY, Operators commuting with translations by one. Part I, Representation theorems, J. Math. Anal. Appl. 4 (1962) 366-410.

[6] D. McGarvey, Operators commuting with translations by one. Part II, Differential operators with periodic coefficients in $L_{p}(-\infty, \infty)$, J. Math. Anal. Appl. 11 (1965) 564-596.

[7] D. McGarvey, Operators commuting with translations by one. Part III, Perturbation results for periodic differential operators, J. Math. Anal. Appl. 12 (1965) 187-234.

[8] F. S. RofE-BEKETOV, The spectrum of nonselfadjoint differential operators with periodic coefficients, Soviet Math. Dokl. 4 (1963) 1563-1566.

[9] V. A. TKACHENKO, Spectral analysis of nonselfadjoint Schrodinger operator with a periodic complex potential, Soviet Math. Dokl. 5 (1964), 413-415.

[10] O. A. VELIEV, The spectrum and spectral singularities of differential operators with complex-valued periodic coefficients, Differential cprime nye Uravneniya 19 (1983), 1316-1324.

[11] O. A. Veliev, M. Toppamuk Duman, The spectral expansion for a nonself-adjoint Hill operators with a locally integrable potential, J. Math. Anal. Appl. 265, (2002) 76-90.

[12] O. A. Veliev, Asymptotic Analysis of Non-self-adjoint Hill Operators, Cent. Eur. J. Math., Volume 11, Issue 12 (2013) pp. 2234-2256.

[13] O. A. Veliev, Spectral Analysis of the Non-self-adjoint Mathieu-Hill Operator, 2013, arXiv:1202.4735v4 